\title{
Monodisperse cluster crystals: Classical and quantum dynamics
}

\author{
Rogelio Díaz-Méndez, ${ }^{1}$ Fabio Mezzacapo, ${ }^{1}$ Fabio Cinti, ${ }^{2}$ Wolfgang Lechner, ${ }^{3}$ and Guido Pupillo ${ }^{1}$ \\ ${ }^{1}$ IPCMS (UMR 7504) and ISIS (UMR 7006), Université de Strasbourg and CNRS, 67000 Strasbourg, France \\ ${ }^{2}$ National Institute for Theoretical Physics, Stellenbosch 7600, South Africa \\ ${ }^{3}$ IQOQI and Institute for Theoretical Physics, University of Innsbruck, 6020 Innsbruck, Austria
}

(Received 26 August 2015; published 9 November 2015)

\begin{abstract}
We study the phases and dynamics of a gas of monodisperse particles interacting via soft-core potentials in two spatial dimensions, which is of interest for soft-matter colloidal systems and quantum atomic gases. Using exact theoretical methods, we demonstrate that the equilibrium low-temperature classical phase simultaneously breaks continuous translational symmetry and dynamic space-time homogeneity, whose absence is usually associated with out-of-equilibrium glassy phenomena. This results in an exotic self-assembled cluster crystal with coexisting liquidlike long-time dynamical properties, which corresponds to a classical analog of supersolid behavior. We demonstrate that the effects of quantum fluctuations and bosonic statistics on cluster-glassy crystals are separate and competing: Zero-point motion tends to destabilize crystalline order, which can be restored by bosonic statistics.
\end{abstract}

DOI: 10.1103/PhysRevE.92.052307

PACS number(s): 64.70.P-, 67.85.-d, 32.80.Ee, 67.10.Fj

\section{INTRODUCTION}

The discovery of novel phases of matter as a result of broken symmetries is of main interest in condensed matter. In quantum physics, a key example is the supersolid phase for bosonic particles [1-3], where the rare simultaneous breaking of two symmetries (i.e., continuous translational and global gauge symmetry) leads to the coexistence of both crystalline and superfluid properties [4-11]. Such a state of matter may be realized for a monodisperse ensemble of particles with clusterforming interactions, at sufficiently low temperatures [7,9].

A phase that does not fall into the scheme of broken symmetries is the glass phase, both classical and quantum, which is a nonequilibrium and disordered, yet stable, phase $[12,13]$. In analogy to the broken-symmetry picture, however, the glass transition is often associated with breaking of space-time homogeneity, or dynamic heterogeneity. The latter is a result of frustration effects, known as self-caging [14], and corresponds to a relaxation that is fast on a local scale and exponentially slow on large ones [15-21]. The search for novel mechanisms for caging and glass effects in both the classical [22] and quantum [23-28] regimes is of central interest in condensed matter as well as atomic and molecular physics $[29,30]$. Interestingly, several works have pointed out that mixtures of cluster-forming particles out-of-equilibrium may realize glassy phases in the classical regime [22,23]. This opens the way to understand the relation between the equilibrium and nonequilibrium properties of classical clusterforming particles compared to their quantum counterparts [22] and thus to investigate the classical to quantum transition in these systems. Indeed, a key question is whether glassiness and supersolidity may be related at a fundamental level.

In this work we analyze the classical equilibrium phases, and the effects of quantum fluctuations and bosonic statistics on such phases, in a two-dimensional model system of ultrasoft particles that, in the quantum regime, has been shown to display the existence of a bosonic cluster supersolid [6-9]. In such a state of matter superfluidity emerges in the presence of a self-assembled cluster-crystalline structure. Here we focus on the classical counterpart of this quantum phase and investigate in detail both its static and dynamical properties using exact theoretical techniques that are valid in the classical and semiclassical regimes.

Specifically, we demonstrate theoretically the following. (i) The low-temperature equilibrium classical phase is an exotic ordered cluster crystal with a dynamical separation between intracluster particle motion and intercluster hopping, mimicking dynamic heterogeneity at equilibrium. This is microscopically due to caging effects at the level of individual clusters, which result in the coexistence of crystalline and liquidlike properties, such as linear particle diffusion as a function of time $t$. This clarifies in what sense this thermodynamic phase is the classical version of a cluster supersolid. (ii) By means of a combination of numerically exact semiclassical and fully quantum techniques, we elucidate the different effects of quantum fluctuations and quantum statistics on the phenomena described here. Surprisingly, we find that these are competing: Zero-point motion tends to destabilize the cluster crystal in favor of liquidlike phases, while bosonic quantum statistics can have the opposite effect of enhancing crystalline behavior. The latter effect is at odds with results for systems such as $\mathrm{He}^{4}$ or dipolar crystals [27], where bosonic statistics always favors a liquid behavior, while the former should be compared to recent findings for polydispersed hard spheres [23,28,31], where quantum fluctuations may help crystallization. Some of these effects may be relevant for systems as diverse as colloidal particles $[22,32,33]$ as well as cold gases of Rydberg atoms [34], where light-dressing techniques can be used to tune effective interparticle interactions [35,36].

The remainder of this paper is organized as follows. In Sec. II we present the Hamiltonian of interest for an ensemble of particles interacting via ultrasoft cluster-forming potentials and summarize known results in the quantum regime. In Sec. III we present the computed classical phase diagram for our model by introducing both static and dynamical order parameters that allow us to characterize the classical counterpart of the quantum cluster supersolid investigated in Ref. [9]. In Sec. IV we introduce exact results for the semiclassical real-time dynamics of our model, by excluding 
particle exchanges, as well as exact results for static properties that include the effects of bosonic quantum statistics. In this way, namely, by understanding the separate effect of zero-point motion and bosonic quantum statistics on the classical phase, we start elucidating the transition between the classical and quantum scenarios. Finally, we outline the conclusions as well as possible extensions of the present work.

\section{HAMILTONIAN: ULTRASOFT PARTICLES}

We consider a two-dimensional ensemble of $N$ bosonic particles with mass $m$, density $\rho$, and Hamiltonian

$$
\hat{H}=-\frac{\hbar^{2}}{2 m} \sum_{i=1}^{N} \nabla_{i}^{2}+\sum_{i<j}^{N} \frac{V_{0}}{r_{i j}^{\gamma}+R_{\mathrm{c}}^{\gamma}} .
$$

The interaction in Eq. (1) approaches a constant value $V_{0} / R_{\mathrm{c}}^{\gamma}$ as the interparticle distance $r$ decreases below the soft-core distance $R_{\mathrm{c}}$ and drops to zero for $r>R_{\mathrm{c}}$. The case $\gamma \rightarrow$ $\infty$ yields the soft-disk model [37]. Here we focus on $\gamma=$ 6 , corresponding to soft-core van der Waals interactions of relevance for ultracold atoms [6,38].

Particles with soft-core interactions have been studied previously [39-43] in the classical high-temperature regime $(\hbar=0, T \neq 0)$ and in the purely quantum zero-temperature regime $(\hbar \neq 0, T=0)[6,7,9]$. In the former case, it has been shown that pair potentials with a negative Fourier component [40] favor the formation of particle clusters, which in turn can crystallize to form so-called classical cluster crystals. While cluster formation has been intensively investigated in the context of, e.g., colloids [44], it has been demonstrated that bosonic quantum statistics can turn a cluster-solid phase into a supersolid phase via a quantum phase transition at a critical value $\alpha_{c s \text {-ss }} \simeq 40$ where $\rho m V_{0} /\left(\hbar^{2} R_{c}^{2}\right) \equiv \alpha$ [9]. The supersolid further melts into a superfluid phase via a first-order quantum phase transition at $\alpha_{s s-s f} \simeq 30$.

Here we bridge the gap between the classical and quantum regimes by first analyzing the static and dynamic properties at the classical level and then studying the separate effects of quantum fluctuations and statistics. We performed our theoretical investigation by using different exact numerical approaches that are appropriate for the various regimes of our interest. Specifically, Langevin molecular dynamics [45] has been employed to obtain the classical phase diagram of model (1). Quantum effects on the classical phases have been studied by means of path-integral molecular dynamics in the semiclassical regime [31] and full path-integral quantum Monte Carlo simulations [46]. The former allows for investigating the real-time dynamics of quantum particles in the absence of quantum statistics, while the latter provides exact results for static properties of bosonic quantum systems.

\section{CLASSICAL PHASE DIAGRAM}

\section{A. Static properties}

The classical $(\hbar=0, T \neq 0)$ phases of model $(1)$, as obtained from Langevin molecular dynamics [45], have been characterized by analyzing both static and dynamical physical observables for systems comprising up to $N=3120$ particles in a wide range of temperatures and densities. Here

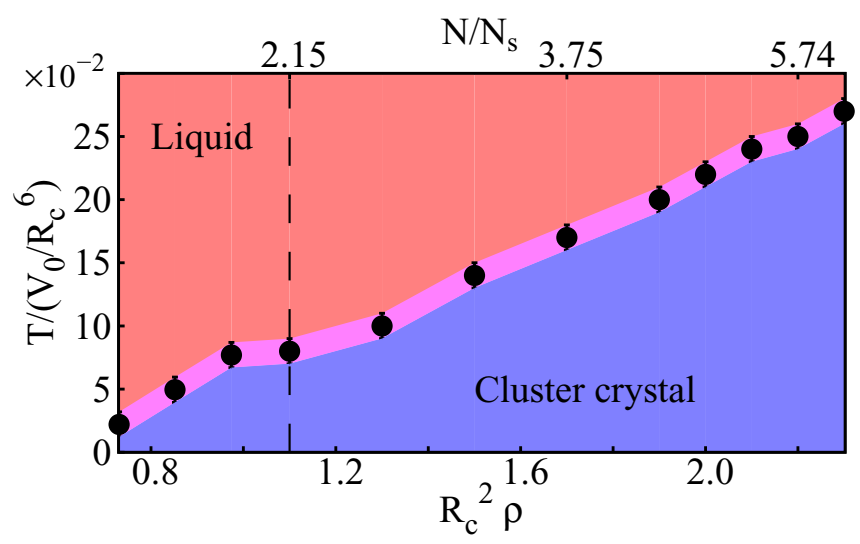

FIG. 1. (Color online) Phase diagram as a function of scaled temperature $T /\left(V_{0} / R_{c}^{6}\right)$ and density $R_{c}^{2} \rho$ for classical monodisperse particles with soft-core interactions [see Eq. (1)] at equilibrium. Here $N / N_{s}$ is the average number of particles per cluster in the ground state (upper $x$ axis). The dashed vertical line marks $R_{c}^{2} \rho \simeq 1.1$ (see Fig. 2).

thermodynamic equilibrium has been achieved by means of a standard annealing procedure. The resulting equilibrium phase diagram is shown in Fig. 1 as a function of temperature $T /\left(V_{0} / R_{c}^{6}\right)$ and the rescaled density $R_{c}^{2} \rho \gtrsim 1$. At low $T$ we find an ordered crystalline phase consisting of clusters arranged in a triangular configuration, each cluster comprising an average number of particles larger than one (see, e.g., Figs. 4 and 7) and increasing with $R_{c}^{2} \rho$. Long-range positional order is first measured by estimating the static structure factor

$$
S(\mathbf{k})=\frac{1}{N}\left\langle\left|\sum_{j}^{N} e^{i \mathbf{k} \cdot \mathbf{r}_{j}}\right|^{q}\right\rangle .
$$

Here $\mathbf{k}$ is a wave vector, $\mathbf{r}_{j}$ is the position of the $j$ th particle, and $\langle\cdots\rangle$ denotes averaging over many configurations. This observable displays well-defined peaks in the low- $T$ ordered phase [Fig. 2, inset (a)], while, as expected, for high $T$ we

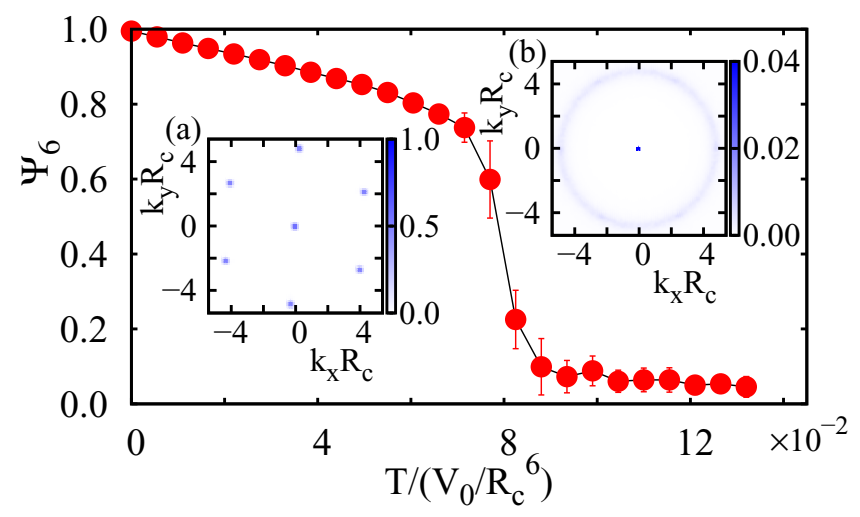

FIG. 2. (Color online) Cluster short-range orientational order $\Psi_{6}$ as a function of $T$ at equilibrium for $R_{c}^{2} \rho \simeq 1.1$. The insets show the static structure factor $S(\mathbf{k})$ at the same rescaled density for (a) $T /\left(V_{0} / R_{c}^{6}\right)=0.02$ and (b) $T /\left(V_{0} / R_{c}^{6}\right)=0.09$. In the former case the system is a cluster crystal, in the latter, as indicated by the extremely small value of $\Psi_{6}$ and the essentially featureless $S(\mathbf{k})$, a liquid. 
find a normal liquid phase with no peaks in $S(\mathbf{k})$ for $\mathbf{k} \neq 0$, independently of $R_{c}^{2} \rho$ [Fig. 2, inset (b)].

We then characterize quantitatively the finite- $T$ melting transition between the cluster crystal and the normal liquid phase by monitoring the hexatic (short-range) bond-order parameter of the clusters, which, in analogy to regular noncluster-forming crystals (see [47] and the Appendix for details), we define as

$$
\Psi_{6}=\frac{1}{N_{c} N_{j}}\left\langle\left|\sum_{j}^{N_{c}} \sum_{l}^{N_{j}} e^{i 6 \theta_{j l}}\right|\right\rangle .
$$

Here $N_{c}$ is the total number of clusters, $N_{j}$ is the number of clusters neighboring the $j$ th one, and $\theta_{j l}$ is the angle between a reference axis and the segment joining the clusters $j$ and $l$ (Fig. 7 in the Appendix). The parameter $\Psi_{6}(T)$ decreases from $\Psi_{6}=1$ at $T=0$ to $\Psi_{6} \simeq 0$ in the liquid phase and displays a sudden jump at the transition point (see the main panel of Fig. 2 for an example). This observed jump is system-size independent for $N \gtrsim 200$ and consistent with a first-order transition. We find that for $R_{c}^{2} \rho \gtrsim 1$ the classical melting temperature $T_{\mathrm{M}}$ grows essentially linearly with $R_{c}^{2} \rho$, with a scaling of the critical interaction strength $\alpha_{c c-l} \equiv \rho V_{0} /\left(T_{\mathrm{M}} R_{c}^{4}\right) \simeq 0.16$.

The precise measurement of $\Psi_{6}$ allows us to obtain the complete phase diagram of Fig. 1 by monitoring static properties within each phase. In the next section we show that dynamical observables are necessary in order to fully characterize the various phases of the model at equilibrium.

\section{B. Dynamical properties}

The dynamics of the equilibrium phases of Fig. 1 is instead initially characterized by computing the mean square displacement

$$
\operatorname{MSD}(t)=\left\langle\Delta r^{2}(t)\right\rangle=\frac{1}{N}\left\langle\sum_{j}\left|\mathbf{r}_{j}(0)-\mathbf{r}_{j}(t)\right|^{2}\right\rangle,
$$

as well as the time-dependent self-intermediate scatter function

$$
F_{S}\left(k^{*}, t\right)=\frac{1}{N}\left\langle\sum_{j} e^{i k^{*}\left[\mathbf{r}_{j}(0)-\mathbf{r}_{j}(t)\right]}\right\rangle .
$$

Here $k^{*}=\left|\mathbf{k}^{*}\right|$ refers to the characteristic wave vector of the main peak in $S(\mathbf{k})$ [29]. These quantities provide complementary information on particle mobility and time correlations of particle positions within the crystal, respectively [49]: For example, in the liquid phase, $\operatorname{MSD}(t)$ follows the linear diffusion law $\operatorname{MSD}(t) \propto t$ [50] typical of Brownian motion, while $F_{s}\left(k^{*}, t\right)$ decays exponentially.

For model (1) the situation is however strikingly different. For $T \lesssim T_{\mathrm{M}}$ the time evolution of both observables, shown in Figs. 3(a) and 3(b), interpolates between the solid and liquid regimes: An extended plateau is followed by linear diffusion for $\operatorname{MSD}(t)$ and exponential decay for $F_{s}\left(k^{*}, t\right)$, respectively, with the size of the plateaus increasing with decreasing $T$. We find that an analysis of the $T$-dependent relaxation time $\tau_{\alpha}$ for which $F_{s}\left(k^{*}, \tau_{\alpha}\right)=1 / e$ reveals an Arrhenius-type exponential dependence on $1 / T$ without detectable saturation [see, e.g.,
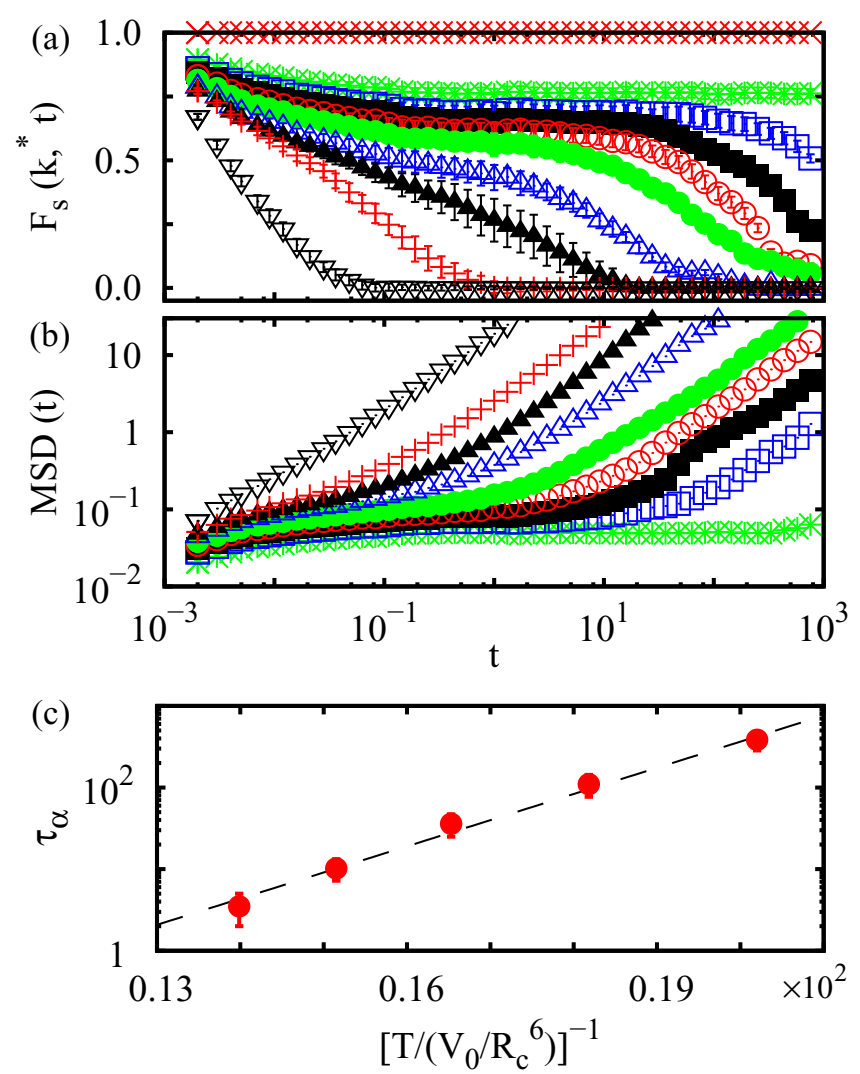

FIG. 3. (Color online) (a) Self-intermediate scatter function $F_{s}\left(k^{*}, t\right)$ vs time $t$ for $T /\left(V_{0} / R_{c}^{6}\right) \times 10^{2}=0,3.3,4.4,4.95,5.5$, $6.05,7.15,7.70,8.25$, and 13.2 (top to bottom). (b) Mean square displacement (in units of $R_{c}^{2}$ ) $\operatorname{MSD}(t)$ vs $t$; symbols are the same as in (a). (c) Relaxation time $\tau_{\alpha}$ defined via $F_{s}\left(k^{*}, \tau_{\alpha}\right)=e^{-1}$. The dashed line is an exponential fitting function (see the text). Data refer to the scaled density $R_{c}^{2} \rho=1.1$.

Fig. 3(c)]. This indicates that the liquidlike diffusion described here is thermally activated and only vanishes at $T=0$.

These results are interesting as the phenomenology reported here, that is, the existence of plateaus in the time evolution of $\operatorname{MSD}(t)$ and $F_{s}\left(k^{*}, t\right)$, is typical of, e.g., glass-forming liquids. In particular, the Arrhenius-type dynamics would usually correspond to a peculiar strong-glass behavior. This immediately calls for an explanation of the corresponding microscopic dynamics responsible for this macroscopic behavior.

By inspection of particle configurations, we determine that the microscopic diffusion mechanism here corresponds to hopping of particles between different clusters, leaving the underlying crystal structure essentially unaltered. Examples of this dynamics are given in the snapshots of Fig. 4. These behaviors indicate the existence of liquidlike particle diffusion within the crystalline phase at equilibrium. Specifically, for the case of our interest, namely, $R_{c}^{2} \rho>1$, this hopping mechanism occurs in the presence of bond orientational order of the clusters for any temperature within the interval $0 \lesssim T \lesssim T_{\mathrm{M}}$.

We further characterize the dynamical properties of our system by estimating the following non-Gaussian parameter 


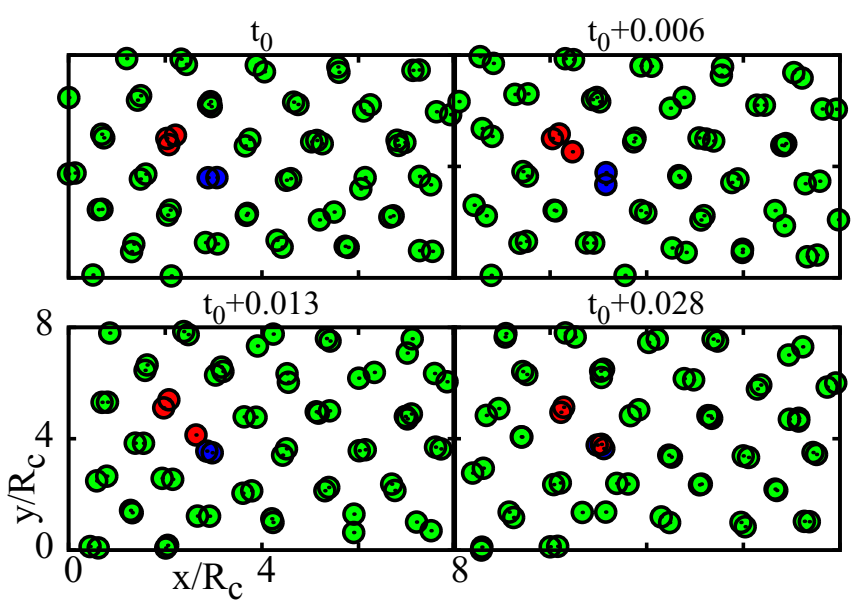

FIG. 4. (Color online) Snapshots of the sequential evolution of a portion of the system, starting at $t=t_{0}$. Particles belonging to sites involved in a hopping process have been highlighted in red and blue.

$[14,51]$

$$
\alpha_{2}(t)=\left[\frac{\left\langle\Delta r^{4}(t)\right\rangle}{2\left\langle\Delta r^{2}(t)\right\rangle^{2}}\right]-1 .
$$

The latter measures deviations from Gaussian fluctuations in the distributions of displacements and thus is in general $\alpha_{2}(t) \simeq 0$ for all $t$ in regular liquids and noncluster crystals at equilibrium. Here, however, for $T<T_{\mathrm{M}}$ and intermediate times we obtain $\alpha_{2}(t) \neq 0$ since the particles can be differentiated into fast and slow due to deconfined intercluster hopping and confined intracluster motion. This is demonstrated for our model in Fig. 5, where, as before, all simulations have been performed at equilibrium. The presence of a peak in the non-Gaussian parameter mimics, in our model, the socalled dynamic heterogeneity usually associated with glassy dynamics where different time scales emerge.

We note that some of the general phenomena described above, such as the deviation from the Gaussian distribution

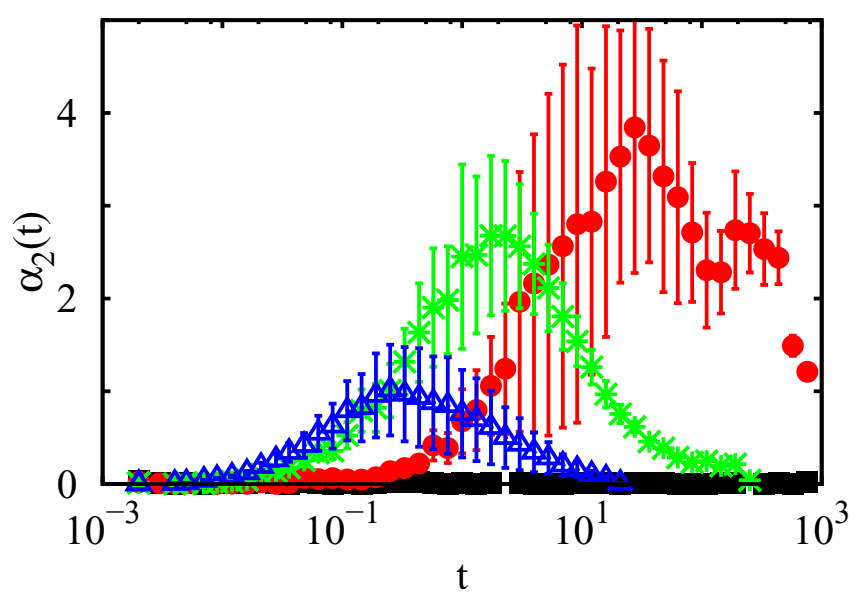

FIG. 5. (Color online) Non-Gaussian parameter $\alpha_{2}(t)$ vs $t$ for $T /\left(V_{0} / R_{c}^{6}\right)=0.03$ (black squares), 0.05 (red circles), 0.07 (green stars), and 0.08 (blue triangles). Data refer to the scaled density $R_{c}^{2} \rho=1.1$. in the fluctuations of displacements, are characteristic of all cluster-forming models and have been recently observed numerically in three-dimensional situations of relevance to soft matter [42]. In addition to analyzing the macroscopic order parameters and the specific microscopic realization of these effects in a two-dimensional model of direct experimental interest for low-temperature atomic physics, here we explain how (and in what sense) these cluster-forming crystals correspond to the classical counterpart of the recently discovered cluster supersolids in the quantum regime. In fact, although the existence of a high-temperature crystalline phase related to the supersolid phase was discussed previously [7,9], its explanation in terms of dynamical properties is one of the main results of this work.

In the following, we start bridging the gap between these classical and quantum regimes, by investigating quantum effects in the semiclassical and fully quantum cases, at equilibrium. Specifically, we show that while zero-point motion results in an enhancement of liquidlike properties, bosonic quantum statistics favors crystalline ones. The analysis of these separate competing roles is crucial to understanding how the supersolid phase emerges from its classical analog.

\section{QUANTUM EFFECTS}

Quantum effects $(\hbar \neq 0, T>0)$ on the phase diagram of Fig. 1 are investigated numerically in the semiclassical approximation using path-integral Langevin dynamics (PIMD) [31] and fully quantum mechanically using exact quantum path-integral Monte Carlo (PIMC) methods [46]. These provide complementary information: PIMD neglects particle exchange and the classical limit is the real-time dynamics of the particles, while the PIMC method treats the bosonic statistics exactly in imaginary time.

\section{A. Semiclassical dynamics}

Figure 6(a) shows results for $F_{s}\left(k^{*}, t\right)$ defined above using PIMD. We choose here scaled densities such as $\alpha \gtrsim \alpha_{c s-s s}$, so that the zero-temperature quantum phase is a cluster crystal even in the quantum regime. In full generality, Fig. 6 shows that semiclassical quantum fluctuations (e.g., zero-point motion) cooperate with thermal fluctuations to enhance local mobility. At low $T$, this effect tends to destabilize crystalline order.

Indeed, fluctuations can affect the equilibrium glassylike dynamics discussed above leading to melting into a liquid phase. As an example, the figure shows that for $R_{c}^{2} \rho=0.8119$ and $T=2.6$ [in units of $\hbar^{2} /\left(m R_{c}^{2}\right)$ ] the relaxation time sensibly decreases when the interaction strength is lowered from $m V_{0} /\left(\hbar^{2} R_{c}^{4}\right)=80$ to $m V_{0} /\left(\hbar^{2} R_{c}^{4}\right)=60$. These semiclassical results should be compared to those of Ref. [31] for a gas of polydispersed hard-sphere liquids, where for certain parameters quantum fluctuations may induce an increase of glassiness, and thus a reentrant behavior, before melting.

\section{B. Effects of bosonic statistics}

The effects of bosonic quantum statistics are shown in Fig. 6(b). There we present results for the radial 

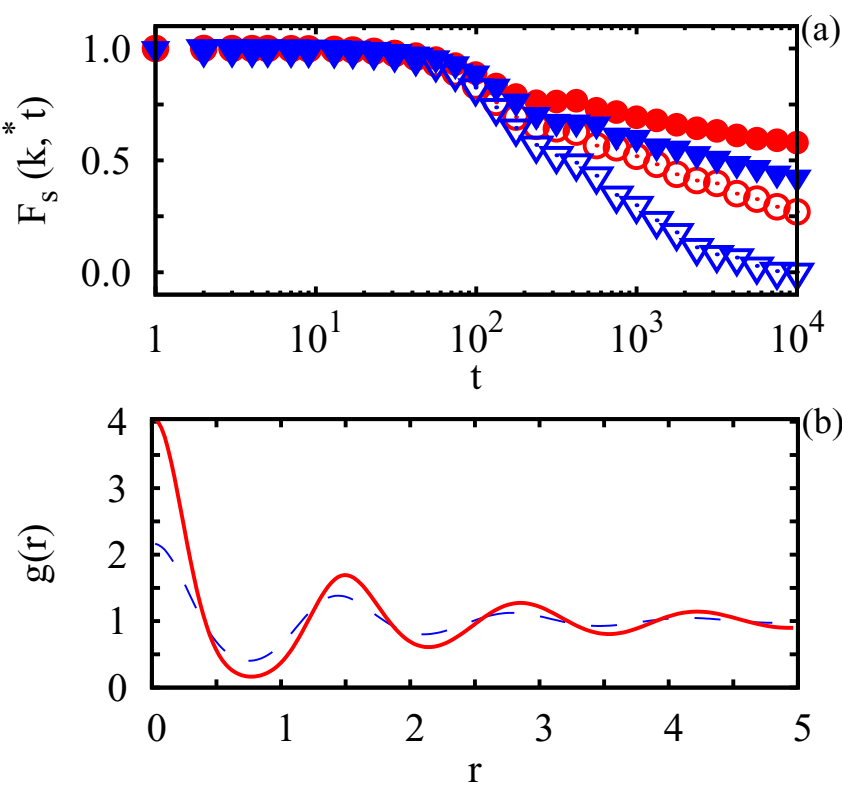

FIG. 6. (Color online) (a) Function $F_{s}\left(k^{*}, t\right)$ computed by pathintegral Langevin dynamics. Data are for $R_{c}^{2} \rho=0.8119$ and $m V_{0} /\left(\hbar^{2} R_{c}^{4}\right)=80$ (circles) and 60 (triangles). The $T$ values are 1.6 and 2.6 (full and empty symbols, respectively), in units of $\hbar^{2} /\left(m R_{c}^{2}\right)$. (b) Function $g(r)$ computed via path-integral Monte Carlo simulations with and without bosonic quantum exchanges (solid and dashed lines, respectively). Here $T /\left[\hbar^{2} /\left(m R_{c}^{2}\right)\right]=10, m V_{0} /\left(\hbar^{2} R_{c}^{4}\right)=35$, and $R_{c}^{2} \rho=2.029$.

density-density correlation function defined as

$$
g(r)=\frac{1}{N}\left\langle\sum_{j}\left[\delta n_{j} /(2 \pi r \delta r)\right]\right\rangle,
$$

where $\delta n_{j}$ is the number of particles at a distance between $r$ and $r+\delta r$ from particle $j$. Here $g(r)$ is computed for Boltzmann particles (i.e., no particle exchanges, dashed line) as well as for Bose particles (i.e., including bosonic statistics, solid line) [52]. As an example, we choose $R_{c}^{2} \rho=2.029, m V_{0} /\left(\hbar^{2} R_{c}^{4}\right)=$ 35 , and $T /\left[\hbar^{2} /\left(m R_{c}^{2}\right)\right]=10$.

The figure shows that the density-density correlations in the semiclassical case are liquidlike. In particular, oscillations of $g(r)$ beyond the first correlation shell are strongly damped and the value of $g(0)$ is approximately 2 . However, this latter value increases by a factor of about 2 when quantum statistics is taken into account (solid line), i.e., in a fully quantum mechanical calculation. In addition, $g(r)$ displays more pronounced oscillations at finite $r$, signaling the enhancement of solidlike behavior. While less efficient for $R_{c}^{2} \rho<1$, we find that this enhancement of solidlike properties is a general feature at sufficiently high density $\left(R_{c}^{2} \rho \gtrsim 1\right)$ [53]. This is in contrast to the physics of noncluster crystals, such as purely dipolar bosons [54,55] or $\mathrm{He}^{4}$ [2], where, as shown in Ref. [27], bosonic statistics always enhances superfluid properties.

In summary, from the analysis above we conclude that, surprisingly, the effects of quantum fluctuations and statistics can be competing: Zero-point motion tends to destabilize the cluster crystal in favor of liquidlike phases, while bosonic quantum statistics can have the opposite effect of enhancing crystalline behavior. Since PIMC simulations (as well as essentially any other methods) do not allow for the investigation of the real-time evolution of the interacting many-body problem in the presence of quantum exchanges, it remains an open question to determine the precise dynamics of these systems in the quantum regime.

\section{CONCLUSION AND OUTLOOK}

We have demonstrated that a two-dimensional model of monodisperse cluster forming particles can realize a classical equilibrium phase that simultaneously breaks both translational symmetry and dynamic homogeneity. While the latter phenomenon is usually associated with out-of-equilibrium glassy physics, here we find it at equilibrium. This results in the realization of a classical self-assembled cluster crystal with coexisting liquidlike properties. This corresponds to a classical analog of the quantum mechanical supersolid phase of matter.

The coexistence of a cluster crystalline structure and of particle diffusion has been explained here in terms of a thermally activated hopping mechanism, where particles delocalize without altering the underlying cluster crystalline matrix. In addition, we have determined the competing effects that quantum mechanical fluctuations and statistics produce on a classical cluster crystal. As ultrasoft interactions are now observable in experiments with Rydberg atoms, this work may open up the exciting possibility of observing soft-matter phenomena in the classical and quantum regimes in atomic physics. Furthermore, interesting theoretical questions are still open, including whether out-of-equilibrium quench dynamics can suppress the structural order presented in this work and what would be the resulting classical and quantum phases.

\section{ACKNOWLEDGMENTS}

We thank T. Pohl for discussions. W.L. acknowledges support from the Austrian Science Fund through Grant No. P 25454-N27 and from the Institut für Quantenoptics und Quanteninformation. G.P. acknowledges support from the European Commission via ERC-St grant ColdSIM (No. 307688), EOARD, and UdS via Labex NIE and IdEX, ITN
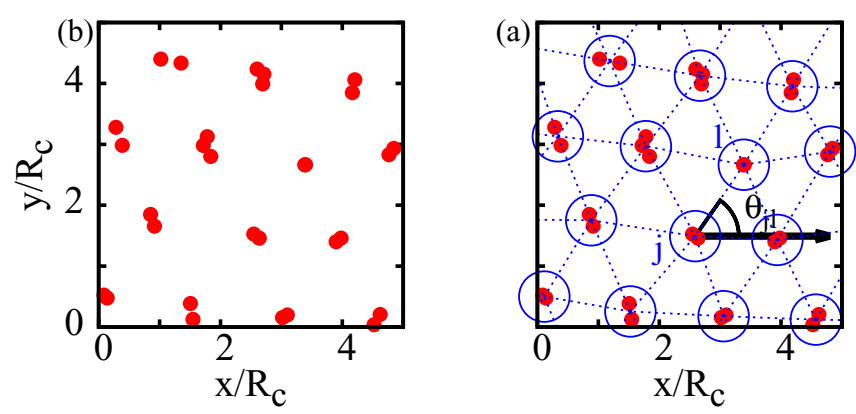

FIG. 7. (Color online) Snapshot of a portion of the system in a cluster crystal configuration. (a) Individual particles. (b) Schematic outcome after the hierarchical clustering technique: Blue circles are centered in cluster centroids, dashed blue segments connect nearestneighbor clusters, and $\theta_{j l}$ is the angle between the $x$ axis and the line joining cluster $j$ with its neighbor $l$. 
COHERENCE, RYSQ, and computing time at the HPC-UdS, ANR through "BLUESHIELD."

\section{APPENDIX: CLUSTERING TECHNIQUE}

In order to calculate the hexatic (short-range) order of the cluster crystal the first step is to distinguish between different clusters. Here we use a hierarchical clustering technique [56] that associates each particle to a single cluster in an unambiguous way (Fig. 7 and text below).

For a given configuration of the system, the algorithm starts with $N_{c}=N$ one-particle clusters, corresponding to the $N$ single particles and their positions. Then an iterative step consists in finding the minimum distance between all pair of clusters, in order to merge the two nearest clusters into a single one, and in relabeling the corresponding particles. The position of the new cluster (formed by the union of the previous two) is defined as the centroid of all the associated particles. The procedure ends when the minimum distance between pairs of clusters is greater than a fixed number $d_{c}$. The value of $d_{c}$ has been set to $d_{c}=0.7 R_{c}$ in our calculations, roughly corresponding to half the value of the first peak in the density-density correlation function. This peak remains at the same position for all densities.
[1] A. B. Kuklov, N. V. Prokof'ev, and B. V. Svistunov, Physics 4, 109 (2011).

[2] M. Boninsegni and N. V. Prokof'ev, Rev. Mod. Phys. 84, 759 (2012).

[3] M. H. W. Chan, R. B. Hallock, and L. Reatto, J. Low Temp. Phys. 172, 317 (2013).

[4] E. P. Gross, Phys. Rev. 106, 161 (1957).

[5] S. Saccani, S. Moroni, and M. Boninsegni, Phys. Rev. Lett. 108, 175301 (2012).

[6] N. Henkel, R. Nath, and T. Pohl, Phys. Rev. Lett. 104, 195302 (2010).

[7] F. Cinti, P. Jain, M. Boninsegni, A. Micheli, P. Zoller, and G. Pupillo, Phys. Rev. Lett. 105, 135301 (2010).

[8] N. Henkel, F. Cinti, P. Jain, G. Pupillo, and T. Pohl, Phys. Rev. Lett. 108, 265301 (2012).

[9] F. Cinti, T. Macrí, W. Lechner, G. Pupillo, and T. Pohl, Nat. Commun. 5, 3235 (2014).

[10] I. Danshita and C. A. R. Sá de Melo, Phys. Rev. Lett. 103, 225301 (2009); L. Pollet, J. D. Picon, H. P. Büchler, and M. Troyer, ibid. 104, 125302 (2010); B. Capogrosso-Sansone, C. Trefzger, M. Lewenstein, P. Zoller, and G. Pupillo, ibid. 104, 125301 (2010).

[11] Y. Li, G. I. Martone, L. P. Pitaevskii, and S. Stringari, Phys. Rev. Lett. 110, 235302 (2013).

[12] M. Merolle, J. P. Garrahan, and D. Chandler, Proc. Natl. Acad. Sci. USA 102, 10837 (2005).

[13] M. S. Shell, P. G. Debenedetti, and F. H. Stillinger, J. Phys.: Condens. Matter 17, S4035 (2005).

[14] P. Charbonneau, A. Ikeda, G. Parisi, and F. Zamponi, Proc. Natl. Acad. Sci. USA 109, 13939 (2012).

[15] K. Binder and W. Kob, Glassy Materials and Disordered Solids (World Scientific, London, 2011).

[16] P. G. Wolynes and V. Lubchenko, Structural Glasses and Supercooled Liquids (Wiley, Hoboken, 2012).

[17] P. G. Debenedetti and F. H. Stillinger, Nature (London) 410, 259 (2001).

[18] G. L. Hunter and E. R. Weeks, Rep. Prog. Phys. 75, 066501 (2012).

[19] F. Sciortino, W. Kob, and P. Tartaglia, Phys. Rev. Lett. 83, 3214 (1999).

[20] W. Götze, J. Phys.: Condens. Matter 11, A1 (1999).
[21] H. Tanaka, T. Kawasaki, H. Shintani, and K. Watanabe, Nat. Mater. 9, 324 (2010).

[22] F. Sciortino and E. Zaccarelli, Nature (London) 493, 30 (2013).

[23] F. Zamponi, Nat. Phys. 7, 99 (2011).

[24] G. Biroli, C. Chamon, and F. Zamponi, Phys. Rev. B 78, 224306 (2008).

[25] Z. Nussinov, Physics 1, 40 (2008).

[26] G. Carleo, M. Tarzia, and F. Zamponi, Phys. Rev. Lett. 103, 215302 (2009).

[27] M. Boninsegni, L. Pollet, N. Prokof'ev, and B. Svistunov, Phys. Rev. Lett. 109, 025302 (2012).

[28] Z. Nussinov, P. Johnson, M. J. Graf, and A. V. Balatsky, Phys. Rev. B 87, 184202 (2013).

[29] W. Lechner and P. Zoller, Phys. Rev. Lett. 111, 185306 (2013).

[30] I. Lesanovsky and J. P. Garrahan, Phys. Rev. Lett. 111, 215305 (2013)

[31] T. E. Markland, J. A. Morrone, B. J. Berne, K. Miyazaki, E. Rabani, and D. R. Reichman, Nature Phys. 7, 134 (2011).

[32] A. Narros, A. J. Moreno, and C. N. Likos, Soft Matter 6, 2435 (2010).

[33] Y. Li, Y. D. Tseng, S. Y. Kwon, L. d'Espaux, J. S. Bunch, P. L. McEuen, and D. Luo, Nat. Mater. 3, 38 (2003).

[34] N. Malossi, M. M. Valado, S. Scotto, P. Huillery, P. Pillet, D. Ciampini, E. Arimondo, and O. Morsch, Phys. Rev. Lett. 113, 023006 (2014); H. Schempp, G. Günter, M. Robertde-Saint-Vincent, C. S. Hofmann, D. Breyel, A. Komnik, D. W. Schnleber, M. Gärttner, J. Evers, S. Whitlock, and M. Weidemüller, ibid. 112, 013002 (2014); D. Barredo, S. Ravets, H. Labuhn, L. Béguin, A. Vernier, F. Nogrette, T. Lahaye, and A. Browaeys, ibid. 112, 183002 (2014); D. Maxwell, D. J. Szwer, D. Paredes-Barato, H. Busche, J. D. Pritchard, A. Gauguet, K. J. Weatherill, M. P. A. Jones, and C. S. Adams, ibid. 110, 103001 (2013); T. Baluktsian, B. Huber, R. Löw, and T. Pfau, ibid. 110, 123001 (2013); M. Viteau, P. Huillery, M. G. Bason, N. Malossi, D. Ciampini, O. Morsch, E. Arimondo, D. Comparat, and P. Pillet, ibid. 109, 053002 (2012); T. Peyronel, O. Firstenberg, Q.-Y. Liang, S. Hofferberth, A. V. Gorshkov, T. Pohl, M. D. Lukin, and V. Vuletic, Nature (London) 488, 57 (2012); P. Schauß, M. Cheneau, M. Endres, T. Fukuhara, S. Hild, A. Omran, T. Pohl, C. Gross, S. Kuhr, and I. Bloch, ibid. 491, 87 (2012). 
[35] L. Santos, G. V. Shlyapnikov, P. Zoller, and M. Lewenstein, Phys. Rev. Lett. 85, 1791 (2000); G. Pupillo, A. Micheli, M. Boninsegni, I. Lesanovsky, and P. Zoller, ibid. 104, 223002 (2010); J. Honer, H. Weimer, T. Pfau, and H. P. Büchler, ibid. 105, 160404 (2010); J. B. Balewski, A. T. Krupp, A. Gaj, S. Hofferberth, R. Löw, and T. Pfau, New J. Phys. 16, 063012 (2014).

[36] Y.-Y. Jau, A. M. Hankin, Tyler Keating, I. H. Deutsch, and G. W. Biedermann, arXiv:1501.03862.

[37] Y. Pomeau and S. Rica, Phys. Rev. Lett. 72, 2426 (1994).

[38] F. Maucher, N. Henkel, M. Saffman, W. Królikowski, S. Skupin, and T. Pohl, Phys. Rev. Lett. 106, 170401 (2011).

[39] C. N. Likos, A. Lang, M. Watzlawek, and H. Löwen, Phys. Rev. E 63, 031206 (2001).

[40] B. M. Mladek, D. Gottwald, G. Kahl, M. Neumann, and C. N. Likos, Phys. Rev. Lett. 96, 045701 (2006).

[41] A. J. Moreno and C. N. Likos, Phys. Rev. Lett. 99, 107801 (2007).

[42] D. Coslovich, L. Strauss, and G. Kahl, Soft Matter 7, 2127 (2011).

[43] M. Montes-Saralegui, A. Nikoubashman, and G. Kahl, J. Phys.: Condens. Matter 25, 195101 (2013).

[44] A. Stradner, H. Sedgwick, F. Cardinaux, W. C. K. Poon, S. U. Egelhaal, and P. Schurtenberger, Nature (London) 432 , 492 (2004).

[45] P. M. Chaikin and T. C. Lubensky, Principles of Condensed Matter Physics (Cambridge University Press, Cambridge, 1995).
[46] M. Boninsegni, N. Prokof'ev, and B. Svistunov, Phys. Rev. Lett. 96, 070601 (2006).

[47] We note that our definition of $\Phi_{6}$ reduces to the standard definition of bond-order parameter for single-particle crystals when $N_{c}=N$ (see, e.g., Ref. [48]). Technical details on the procedure we adopted to define a cluster are given in the Appendix.

[48] N. Gribova, A. Arnold, T. Schilling, and C. Holm, J. Chem. Phys. 135, 054514 (2011).

[49] J.-P. Hansen and I. R. McDonald, Theory of Simple Liquids (Academic, New York, 2013).

[50] K. L. Ngai, Relaxation and Diffusion in Complex Systems (Springer, New York, 2011).

[51] A. Widmer-Cooper, P. Harrowell, and H. Fynewever, Phys. Rev. Lett. 93, 135701 (2004).

[52] Real-time dynamics is not directly accessible using quantum Monte Carlo techniques, which are in turn exact for static observables, e.g., $g(r)$, in the presence of exchanges.

[53] The solid-liquid phase transition becomes essentially density independent for $R_{c}^{2} \rho \gg 1$; see F. Cinti, M. Boninsegni, and T. Pohl, New J. Phys. 16, 033038 (2014).

[54] H. P. Büchler, E. Demler, M. Lukin, A. Micheli, N. Prokof'ev, G. Pupillo, and P. Zoller, Phys. Rev. Lett. 98, 060404 (2007).

[55] G. E. Astrakharchik, J. Boronat, I. L. Kurbakov, and Y. E. Lozovik, Phys. Rev. Lett. 98, 060405 (2007).

[56] P. Tan, M. Steinbach, and V. Kumar, Introduction to Data Mining (Addison-Wesley, Reading, 2006). 